\title{
ON THE THEORY OF THE MATTHEWS AND THE RUSSELL- LÉONARD PHOTOMETERS FOR THE MEASUREMENT OF MEAN SPHERICAL AND MEAN HEMISPHERICAL INTENSITIES.
}

By Edward P. Hyde.

\section{INTRODUCTION.}

Much attention has been given in the last few years to the development of photometers designed for the measurement of mean spherical and mean hemispherical intensities of light sources. Among other instruments of this type that have appeared may be mentioned particularly the two instruments ${ }^{a}$ designed by Matthews, and the more recent instrument ${ }^{b}$ of Léonard, based on theory first given by Russell. ${ }^{c}$ In each of these photometers mirrors are employed in order to project upon the photometer screen light emitted by the source at various inclinations to the vertical axis, but in each instrument a different method is used for weighting the light from each mirror, so that in the summation the light from each mirror shall be diminished in proportion to the area of the zone in which the mirror lies and for which it is supposed to give the mean value.

In the Matthews integrating photometer for incandescent lamps the light from each mirror falls upon the screen at an angle of incidence, $90^{\circ}-\theta,{ }^{d}$ which is the same as the angle between the horizontal direction and the line joining the mirror in question with the light source. The area of a zone of latitude $90^{\circ}-\theta$ is proportional to $\sin \theta$, and the intensity of illumination produced by light incident at angle $90^{\circ}-\theta$ is

$a$ Trans. Amer. Inst. of Elec. Eng., 18, p. 677, 1901; 20, p. 1465, 1902.

${ }^{b}$ L'Éclairage Électrique, 40, p. 128, 1904.

c Jour. Inst. of Elec. Eng., 32, p. 631, 1903.

$d$ In order to make the use of " $\theta$ " in this connection consistent with its subsequent use in the paper, it is necessary to denote the angle of incidence by " $90^{\circ}-\theta$ " instead of by " $\theta . "$ 
cut down in the ratio of $1: \sin \theta$; therefore, the light from each mirror produces an illumination of the screen proportional to the area of the zone in which the mirror lies, and hence receives its proper weight in the summation. In the integrating photometer for are lamps, however, the light from each mirror is incident upon the screen at the same angle, but between the screen and the circle of mirrors is interposed a circular glass disk divided into as many sectors as there are mirrors, and having each sector so smoked that its coefficient of transmission is proportional to the cosine of the angle, $90^{\circ}-\theta$, between the horizontal direction in the plane of the mirrors and the line joining the light-source with the mirror to which the sector in question corresponds. In this way the light from each mirror is cut down in the ratio of $1: \sin \theta$, and therefore receives its proper weight in the summation for the mean spherical intensity.

It is to be especially noted that in the theory of each of the above instruments the surface of the unit sphere is divided into an integral number of zones, not of equal area, but of equal arc, so that the area of each zone is proportional to $\sin \theta$, and to each zone a mirror is assigned. Suppose, now, that instead of dividing the surface of the sphere into zones of equal arc we divide it into zones of equal area, and in each zone place a mirror in such a position that it gives the mean value of the intensity for the zone in which it lies. Under these circumstances the light from each mirror, since it stands in every case for a zone of the same area, is to be given the same weight in the summation for mean spherical intensity, and consequently no further weighting is necessary.

This principle is made use of in the instrument of Léonard, though it was Russell who first suggested that in the determination of mean spherical and mean hemispherical candle-power by a number of readings at intervals in the vertical plane, the readings be made, not at equal angular intervals, as is ordinarily done, but at intervals corresponding to the middle points of successive zones of equal area, so that the unweighted arithmetical mean of the various readings may be taken as the mean spherical intensity of the source.

It was the original intention of this paper to present a more complete theoretical discussion of the Matthews integrating photometers, particularly of the instrument intended for incandescent lamps and sources of like intensity, as it was in connection with the design of such an instrument for the Bureau of Standards that this investigation was undertaken. Since, however, the Russell-Léonard instrument may be treated in an entirely similar manner, we shall conclude the paper with a brief theoretical discussion of this new type of photo- 
meter, as applied both to arc lamps and to incandescent lamps and sources of like intensity.

In the theoretical discussion of the Matthews integrating photometer for incandescent lamps, as given by Professor Matthews before the American Institute of Electrical Engineers, the assumption is implicitly made that eleven pairs of mirrors placed every $15^{\circ}$ (the two end pairs at $0^{\circ}$ and $180^{\circ}$ being omitted) will yield a value of mean spherical candle-power which will agree with the true integral solution to within a negligibly small error. Earlier, in describing his integrating photometer for arc lamps, Professor Matthews makes a definite statement to this effect, and adduces as evidence values computed from the hypothetical intensity curve

$$
I_{\theta}=I_{o} \sin \theta
$$

Thus, starting with the two equations for the mean spherical candlepower, the exact integral equation

$$
I_{m s}=\frac{1}{2} \int_{0}^{\pi} I_{\theta} \sin \theta d \theta
$$

and the approximate equation

$$
I_{m s}=\frac{\pi}{2 n} \Sigma_{0}^{\pi} I_{\theta} \sin \theta
$$

he computes first the true mean spherical candle-power for the distribution $I_{\theta}=I_{o} \sin \theta$ by integration, as follows:

$$
\begin{aligned}
I_{m s} & =\frac{1}{2} \int_{0}^{\pi} I_{\theta} \sin \theta d \theta=\frac{I_{0}}{2} \int_{0}^{\pi} \sin ^{2} \theta d \theta \\
& =.785 I_{o}=78.5 \text { when } I_{o}=100 .
\end{aligned}
$$

He then computes the approximate value from the expression

$$
I_{m s}=\frac{\pi}{2 n} \Sigma_{o}^{\pi} I_{\theta} \sin \theta=\frac{\pi I_{o}}{2 n} \Sigma_{o}^{\pi} \sin ^{2} \theta
$$

for $n=12$ and $\theta=0^{\circ}, 15^{\circ}, 30^{\circ}, \ldots 180^{\circ}$, obtaining as before

$$
I_{m s}=.785 I_{o}=78.5 \text {. }
$$

As a result of this agreement between the integral and the summational values, the conclusion is drawn that a mirror every $15^{\circ}$ is entirely sufficient to give an approximate value which will differ from the true value by a negligibly small error. Now, although it is perfectly allowable to apply results obtained from a hypothetical intensity 
curve to actual intensity curves agreeing more or less closely with it, it can not be assumed that because the integral and summational values agree quite closely for one form of intensity curve that the same agreement can be expected in the cases of intensity curves of different forms. In fact, as will be shown later, there is an error of over 2 per cent entering when we apply the above summational expression to the intensity curve

$$
I_{\theta}=I_{o} \cos \theta .
$$

In the second place it is interesting to note that the hypothetical intensity curve which happened to be chosen in order to show that eleven mirrors are sufficient to give a summation very close to the integral value, will show equally well that any number of mirrors whatever, arranged at equal angular intervals beginning at $\theta=0^{\circ}$ and ending at $\theta=\pi$ will give absolutely the true result. This follows from the equation

$$
\sum_{m=1}^{m=n} \sin ^{2} \frac{m \pi}{n}=\frac{n}{2}
$$

which can be proved as follows:

In order to get rid of the square, put

Then the sum

$$
\sin ^{2} \frac{m \pi}{n}=\frac{1-\cos \frac{2 m \pi}{n}}{2}=\frac{1}{2}-\frac{1}{2} \cos \frac{2 m \pi}{n}
$$

$$
\sum_{m=1}^{m=n} \sin ^{2} \frac{m \pi}{n}=\frac{n}{2}-\frac{1}{2} \sum_{m=1}^{m=n} \cos \frac{2 m \pi}{n}
$$

For convenience, put $\phi=\frac{2 \pi}{n}$ so that

$$
\cos \frac{2 m \pi}{n}=\cos m \phi
$$

We shall now evaluate

$$
\sum_{m=1}^{m=n} \cos m \phi
$$

Introducing the complex expression

we have

$$
\cos m \phi+i \sin m \phi=e^{i m \phi}
$$

$$
\sum_{m=1}^{m=n} \cos m \phi=\text { real part of } \sum_{m=1}^{m=n} e^{i m \phi}
$$


Now the sum of the geometric series

$$
\begin{aligned}
\sum_{m=1}^{m-n} e^{i m \phi} & =e^{i \phi} \frac{1-e^{i n \phi}}{1-e^{i \phi}} \\
& =(\cos \phi+i \sin \phi) \frac{1-\cos n \phi-i \sin n \phi}{1-\cos \phi-i \sin \phi}
\end{aligned}
$$

the real part of which reduces to

$$
\frac{\cos \phi+\cos n \phi-\cos (n+1) \phi-1}{2(1-\cos \phi)}
$$

If, now, we put for $\phi$ its value, $\frac{2 \pi}{n}$, we obtain

$$
\text { real part of } \sum_{m=1}^{m=n} e^{i m \phi}=\sum_{m=1}^{m=n} \cos \frac{2 m \pi}{n}=0
$$

Therefore,

$$
\sum_{m=1}^{m=n} \sin ^{2} \frac{m \pi}{n}=\frac{n}{2}-\frac{1}{2} \sum_{m=1}^{m=n} \cos \frac{2 m \pi}{n}=\frac{n}{2}
$$

From this it follows that

$$
I_{m s}=\frac{\pi}{2 n} \sum_{m=1}^{m=n} I_{o} \sin ^{2} \frac{m \pi}{n}=\frac{\pi}{2 n} \times \frac{n}{2} I_{o}=.785 I_{o}=78.5
$$

Hence we see that if we should divide the sphere into but two zones, placing a single mirror-pair at $\theta=90^{\circ}$, we would still obtain exactly the true integral value for an intensity curve of the form $I_{\theta}=I_{0} \sin \theta$, so that no definite information with regard to the necessary number of mirrors can be obtained from the consideration of this intensity curve alone.

\section{General Theory.}

We shall now pass on to a more complete discussion of the problem of the number of mirrors and their positions on the arc, with particular reference rather to the integrating photometer for incandescent lamps than to the instrument intended especially for arc lamps, since, as stated above, it was in connection with the design of an instrument of the former type for the Bureau of Standards that these computations were made.

Returning to the two equations for the integral and the approximate summational values of the mean spherical intensity,-

$$
I_{m s}=\frac{1}{2} \int_{0}^{\pi} I_{\theta} \sin \theta d \theta
$$




$$
I_{m s}=\frac{\pi}{2 n} \Sigma_{0}^{\pi} I_{\theta} \sin \theta
$$

to which may be added the corresponding expressions for mean hemispherical intensity,-

$$
\begin{gathered}
I_{m h s}=\int_{0}^{\frac{\pi}{2}} I_{\theta} \sin \theta d \theta \\
I_{m h s}=\frac{\pi}{n} \Sigma_{0}^{\frac{\pi}{2}} I_{\theta} \sin \theta
\end{gathered}
$$

we note that the underlying assumption in passing from the integral to the summational expression is that for each zone, $\frac{\pi}{n}$,

$$
I_{\theta} \sin \theta=\text { constant }
$$

or, what amounts to the same thing, that $\theta_{n}$ has been so chosen for each zone that $I_{\theta_{n}} \sin \theta_{n}$ gives the mean value of $I_{\theta} \sin \theta$ for that zone.

Suppose, then, that from a previous decision with regard to the general dimensions of the instrument to be constructed the number of mirrors is determined, the problem consists in so arranging the mirrors that each mirror produces an illumination of the screen that in a way gives the mean for the respective zone, $\frac{\pi}{n}$, in which it lies. Thus we divide the semicircumference into $n$ equal $\operatorname{arcs}, \frac{\pi}{n}$, and to each arc we assign a mirror. This mirror should be placed in its arc in such a position that, so far as possible, it will produce on the screen the mean illumination of the arc. Of course the arrangement of the mirrors would in general be different for each different intensity curve, but we seek to determine which arrangement of mirrors will most nearly satisfy all the more ordinary forms of intensity curves with which we have to deal in the photometry of incandescent lamps and sources of like intensity, such as the Nernst lamp. Obviously, in general it would be undesirable to place the mirrors at the divisions between successive zones. This, however, is what the arrangement in the Matthews instrument as ordinarily constructed would amount to if we attempted to use it for the determination of mean hemispherical intensities, as the horizontal mirrors would be giving the illumination of the zone $\theta=90^{\circ}$ to $\theta=90^{\circ}-\frac{\pi}{n}$. When used for mean spherical deter- 
minations it would seem that the same objection could be urged, because of the two end mirrors at $\theta=0^{\circ}$ and $\theta=\pi$; but in this case we can assume that each mirror gives the mean value of the zone of which it lies in the center, in which case the illumination produced by each end mirror would have to be divided by 2 , since it represents only a halfzone. But since at this incidence $\left(90^{\circ}\right)$ there is no illumination at all produced by these end mirrors in the hypothetical case of a point source, no error is introduced by the incorrect weighting of the end mirrors, for in all cases the effect is zero. (In fact these end mirrors are left out entirely.)

Let us now take several simple, hypothetical curves which are more or less typical of the actual intensity curves of the more ordinary sources, and let us determine for each the distribution of mirrors which will give the true mean spherical value for that case, supposing the semicircumference divided into 20 zones, as that is the number of mirror-pairs determined upon for the instrument under construction at the Bureau of Standards. Let us then apply the true distribution for each individual curve to the other hypothetical intensity curves under consideration, to see how closely it satisfies them; and, finally, let us see how nearly an equal distribution of mirrors satisfies each case, taking both of the equal distributions (for 20 mirror-pairs) $0^{\circ}, 9^{\circ}$, $18^{\circ} \ldots 180^{\circ}$, and $4 \frac{1}{2} \circ, 13 \frac{1}{2} \circ, 22 \frac{1}{2}{ }^{\circ} \ldots . .175 \frac{1}{2}^{\circ}$.

It may be well to call attention here to the advantages gained by dividing the semicircumference into an even number of arcs, and by avoiding such a use of fractions of an arc as is made in the Matthews instrument unless we assume the mirrors of that instrument to be at the divisions between the consecutive arcs, as was pointed out above. If we divide the semicircumference into an even number of arcs and begin counting whole arcs at $\theta=0^{\circ}$ we shall then have a whole number of arcs between $\theta=0^{\circ}$ and $\theta=90^{\circ}$. The advantage of this is that mean hemispherical as well as mean spherical candle-power readings can be made, if in each arc a mirror is so placed as to give the mean value for that arc. Hence, since we have divided the semicircumference into 20 whole arcs, and have assigned a mirror to each arc, every distribution of mirrors which we shall find in the following investigation will be equally applicable to both mean spherical and mean hemispherical determinations. The equal distribution $4 \frac{1}{2} \circ, 13 \frac{1}{2}{ }^{\circ} \ldots . .175 \frac{1}{2} \circ$ also will be applicable to either mean spherical or mean hemispherical measurements, but the equal distribution $0^{\circ}, 9^{\circ} \ldots 180^{\circ}$, having one mirror at $\theta=90^{\circ}$, will only be applicable to mean spherical measurements for the reasons given above.

It may be urged as a practical objection to omitting the mirror at 
$\theta=90^{\circ}$ that the adaptability of the instrument to horizontal measurements is thus destroyed. There seems, however, to be no reason why an extra mirror-pair could not be placed in the horizontal position, keeping it covered during mean spherical determinations. In fact, this would seem preferable to the constant removal and use of the horizontal mirror-pair of the spherical system.

To return to the consideration of the different intensity curves, if the limited number of mirrors is to give the true integral value for any intensity curve, we must assume that each mirror gives the mean value of $I_{\theta} \sin \theta$ for the arc in which it is placed. We must, therefore, in order to get the true distribution of mirrors, divide the semicircumference into $n$ equal arcs and then find the mean value of $I_{\theta} \sin \theta$ for each arc. If the equation of the intensity curve is

$$
I_{\theta}=f(\theta)
$$

then the mean value of $I_{\theta} \sin \theta$ for an arc, $\frac{\pi}{n}$, is

$$
\begin{aligned}
\text { mean }\left[I_{\theta} \sin \theta\right] & =\frac{\int I_{\theta} \sin \theta d \theta}{\int d \theta}=\frac{\int f(\theta) \sin \theta d \theta}{\frac{\pi}{n}} \\
& =\frac{n}{\pi} \int_{(m-1) \frac{\pi}{n}}^{m \frac{\pi}{n}} f(\theta) \sin \theta d \theta
\end{aligned}
$$

where $m$ indicates the order of the zone beginning at $\theta=0^{\circ}$.

If now we were to take the average of all of these means we should get the same result as the true total integral mean, which is

$$
\text { mean }\left[I_{\theta} \sin \theta\right]=\frac{\int_{0}^{\pi} I_{\theta} \sin \theta d \theta}{\int_{0}^{\pi} d \theta}=\frac{1}{\pi} \int_{0}^{\pi} f(\theta) \sin \theta d \theta
$$

In order, now, to find the angular positions of the mirrors corresponding to the solution, we must evaluate the integral of equation (7) for each zone and then solve for $\theta_{m}$ in the equation

$$
I_{\theta_{m}} \sin \theta_{m}=f\left(\theta_{m}\right) \sin \theta_{m}=\frac{n}{\pi} \int_{(m-1) \frac{\pi}{n}}^{m_{n}^{\frac{\pi}{n}}} f(\theta) \sin \theta d \theta
$$

If we placed the mirrors at the angles found, then for that particular intensity curve we would get the true mean spherical intensity. 
Let us now consider several typical cases:

CASE I.

$$
I_{\theta}=\text { constant }=1
$$

This curve corresponds approximately to the intensity curve of a "flattened oval" or of a "spiral" filament. The integral solution is [eq. (8)]

$$
\text { mean value of } \begin{aligned}
I_{\theta} \sin \theta & =\frac{1}{\pi} \int_{0}^{\pi} f(\theta) \sin \theta d \theta \\
& =\frac{1}{\pi} \int_{0}^{\pi} \sin \theta d \theta \\
& =\frac{2}{\pi}[\cos \theta]_{\frac{\pi}{2}}^{0}=\frac{2}{\pi}=0.6366
\end{aligned}
$$

For 20 mirrors the angular distribution would be [eq. (9)]

$$
\begin{aligned}
I_{\theta_{m}} \sin \theta_{m}=\sin \theta_{m} & =\frac{n}{\pi} \int_{(m-1) \frac{\pi}{n}}^{m \frac{\pi}{n}} f(\theta) \sin \theta d \theta=\frac{20}{\pi} \int_{(m-1) \frac{\pi}{20}}^{m \frac{\pi}{20}} \sin \theta d \theta \\
& =\frac{20}{\pi}[\cos \theta]_{m \frac{\pi}{20}}^{(m-1) \frac{\pi}{20}}
\end{aligned}
$$

from which

$$
\theta_{m}=\sin ^{-1} \frac{20}{\pi}[\cos \theta]_{m \frac{\pi}{20}}^{(m-1) \frac{\pi}{20}}
$$

The solution to this case is given in column III of Table I, which is self-explanatory. In columns I and II are given the two equiangular 
distributions. In columns III, V, and VII are given the angular distributions of mirrors satisfying cases I, II, and III, and in columns IV, VI, and VIII are given the angles through which the mirrors must be shifted from the middle points of the ares in order to give the true results for cases I, II, and III, respectively. Values of angles in the quadrant $\theta=90^{\circ}$ to $\theta=180^{\circ}$ are merely the supplements of those given in the table, and are hence omitted.

CASE II.

$$
I_{\theta}=2 a \sin \theta=\sin \theta
$$

This curve corresponds approximately to the intensity curve of a "hairpin" or "horseshoe" filament. The integral solution [eq. (8)] is

$$
\begin{aligned}
\text { mean }\left[I_{\theta} \sin \theta\right] & =\frac{1}{\pi} \int_{0}^{\pi} f(\theta) \sin \theta d \theta=\frac{1}{\pi} \int_{0}^{\pi} \sin ^{2} \theta d \theta \\
& =\frac{1}{\pi}[\theta-\sin \theta \cos \theta]_{0}^{\frac{\mu}{2}}=\frac{1}{\pi} \times \frac{\pi}{2}=0.5000
\end{aligned}
$$

For 20 mirrors the angular distribution would be [eq. (9)]

(15) $I_{\theta_{m}} \sin \theta_{m}=\sin ^{2} \theta_{m}=\frac{20}{\pi} \int_{(m-1) \frac{\pi}{20}}^{m \frac{\pi}{20}} \sin ^{2} \theta d \theta=\frac{10}{\pi}[\theta-\sin \theta \cos \theta]_{(m-1) \frac{\pi}{20}}^{m \frac{\pi}{20}}$ from which

$$
\theta_{m}=\sin ^{-1}\left\{\frac{10}{\pi}[\theta-\sin \theta \cos \theta]_{(m-1) \frac{\pi}{20}}^{\frac{\pi}{20}}\right\}^{\frac{1}{2}}
$$

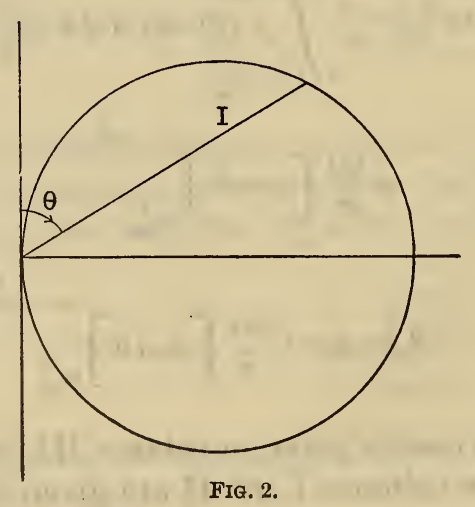


The solution to this case is given in column $\mathrm{V}$ of Table $\mathrm{I}$.

CASE III.

This curve corresponds approximately to the intensity curve of a Nernst or of a "meridian" lamp.

The integral solution [eq. (8)] is

(18) $\operatorname{mean}\left[I_{\theta} \sin \theta\right]=\frac{1}{\pi} \int_{0}^{\pi} f(\theta) \sin \theta d \theta=\frac{1}{\pi} \int_{0}^{\pi} \cos \theta \sin \theta d \theta=\frac{1}{\pi}\left[\sin ^{2} \theta\right]_{0}^{\frac{\pi}{2}}$

$$
=\frac{1}{\pi}=0.3183
$$

For 20 mirrors the angular distribution would be [eq. (9)]

$$
\begin{gathered}
I_{\theta_{m}} \sin \theta_{m}=\sin ^{\circ} \theta_{m} \cos \theta_{m}=\frac{1}{2} \sin 2 \theta_{m}=\frac{20}{\pi} \int_{(m-1) \frac{\pi}{20}}^{m \frac{\pi}{20}} \sin \theta \cos \theta d \theta \\
=\frac{10}{\pi}\left[\sin ^{2} \theta\right]_{(m-1) \frac{\pi}{20}}^{m \frac{\pi}{20}}
\end{gathered}
$$

from which

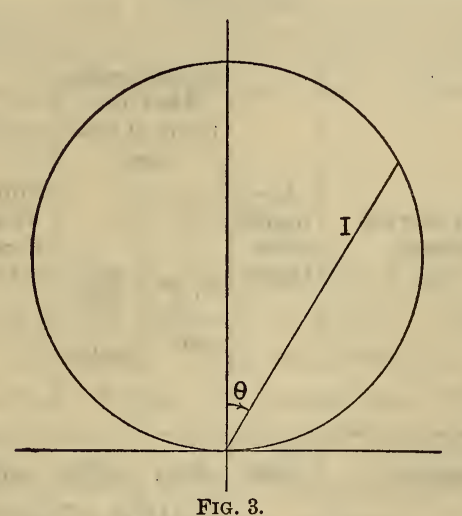

$$
\theta_{m}=\frac{1}{2} \sin ^{-1} \frac{20}{\pi}\left[\sin ^{2} \theta\right]_{(m-1) \frac{\pi}{20}}^{m \frac{\pi}{20}}
$$

The solution to this case is given in column VII of Table I.

Having determined the actual distributions of mirrors for cases I, II, and III, the distribution for each case was tried in the other two cases, and the two equal distributions were also tried in each of the three cases. 
TABLE I.-Various distributions of mirrors.

\begin{tabular}{|c|c|c|c|c|c|c|c|c|c|c|}
\hline \multicolumn{2}{|c|}{$\begin{array}{l}\text { Equiangular } \\
\text { distributions. }\end{array}$} & \multicolumn{2}{|c|}{$\begin{array}{l}\text { Distribu- } \\
\text { tion satisfy } \\
\text { ing case } 1 \text {. } \\
\left(I_{\theta}=1\right)\end{array}$} & \multirow{2}{*}{$\begin{array}{c}\begin{array}{c}\text { Column } \\
\text { III minus } \\
\text { column II. }\end{array} \\
-0^{\prime}\end{array}$} & \multicolumn{2}{|c|}{$\begin{array}{l}\text { Distribu- } \\
\text { tion satisfy- } \\
\text { ing case II. } \\
\left(I_{\theta}=\sin \theta\right)\end{array}$} & \multirow{2}{*}{$\begin{array}{c}\begin{array}{c}\text { Column V } \\
\text { minus } \\
\text { column II. }\end{array} \\
+40^{\prime}\end{array}$} & \multicolumn{2}{|c|}{$\begin{array}{c}\text { Distribu- } \\
\text { tion satisfy- } \\
\text { ing case III. } \\
\left(I_{\theta}=\cos \theta\right)\end{array}$} & \multirow{2}{*}{$\begin{array}{c}\text { Column } \\
\text { vII minus } \\
\text { column II. } \\
-1^{\prime}\end{array}$} \\
\hline $0^{\circ}$ & $4 \frac{1}{2}^{\circ}$ & $4^{\circ}$ & $30^{\prime}$ & & $5^{\circ}$ & $10^{\prime}$ & & $4^{\circ}$ & $29^{\prime}$ & \\
\hline $9^{\circ}$ & $13 \frac{1}{2}^{\circ}$ & $13^{\circ}$ & $29^{\prime}$ & $-1^{\prime}$ & $13^{\circ}$ & $44^{\prime}$ & $+14^{\prime}$ & $13^{\circ}$ & $26^{\prime}$ & $-4^{\prime}$ \\
\hline $18^{\circ}$ & $22 \frac{1}{2}^{\circ}$ & $22^{\circ}$ & $29^{\prime}$ & $-1^{\prime}$ & $22^{\circ}$ & $37^{\prime}$ & $+7^{\prime}$ & $22^{\circ}$ & $23^{\prime}$ & $-7^{\prime}$ \\
\hline $27^{\circ}$ & $31_{2}^{1}{ }^{\circ}$ & $31^{\circ}$ & $28^{\prime}$ & $-2^{\prime}$ & $31^{\circ}$ & $33^{\prime}$ & $+3^{\prime}$ & $31^{\circ}$ & $16^{\prime}$ & $-14^{\prime}$ \\
\hline $36^{\circ}$ & $40 \frac{1}{2}^{\circ}$ & $40^{\circ}$ & $27^{\prime}$ & $-3^{\prime}$ & $40^{\circ}$ & $31^{\prime}$ & $+1^{\prime}$ & $39^{\circ}$ & $49^{\prime}$ & $-41^{\prime}$ \\
\hline $45^{\circ}$ & $49 \frac{1}{2}^{\circ}$ & $49^{\circ}$ & $26^{\prime}$ & $-4^{\prime}$ & $49^{\circ}$ & $29^{\prime}$ & $-1^{\prime}$ & $50^{\circ}$ & $12^{\prime}$ & $+42^{\prime}$ \\
\hline $54^{\circ}$ & $58 \frac{1}{2}^{\circ}$ & $58^{\circ}$ & $24^{\prime}$ & $-6^{\prime}$ & $58^{\circ}$ & $26^{\prime}$ & $-4^{\prime}$ & $58^{\circ}$ & $44^{\prime}$ & $+14^{\prime}$ \\
\hline $63^{\circ}$ & $67 \frac{1}{2}^{\circ}$ & $67^{\circ}$ & $21^{\prime}$ & $-9^{\prime}$ & $67^{\circ}$ & $23^{\prime}$ & $-7^{\prime}$ & $67^{\circ}$ & $37^{\prime}$ & $+7^{\prime}$ \\
\hline $72^{\circ}$ & $76 \frac{1}{2}^{\circ}$ & $76^{\circ}$ & $16^{\prime}$ & $-14^{\prime}$ & $76^{\circ}$ & $16^{\prime}$ & $-14^{\prime \circ}$ & $76^{\circ}$ & $34^{\prime}$ & $+4^{\prime}$ \\
\hline $81^{\circ}$ & $85 \frac{1}{2}^{\circ}$ & $84^{\circ}$ & $48^{\prime}$ & $-42^{\prime}$ & $84^{\circ}$ & $50^{\prime}$ & $-40^{\prime}$ & $85^{\circ}$ & $31^{\prime}$ & $+1^{\prime}$ \\
\hline $90^{\circ}$ & & & & & & & & & & •...... \\
\hline
\end{tabular}

TABLE II.-Results obtained by applying the various distributions of mirrors to each of the three cases.

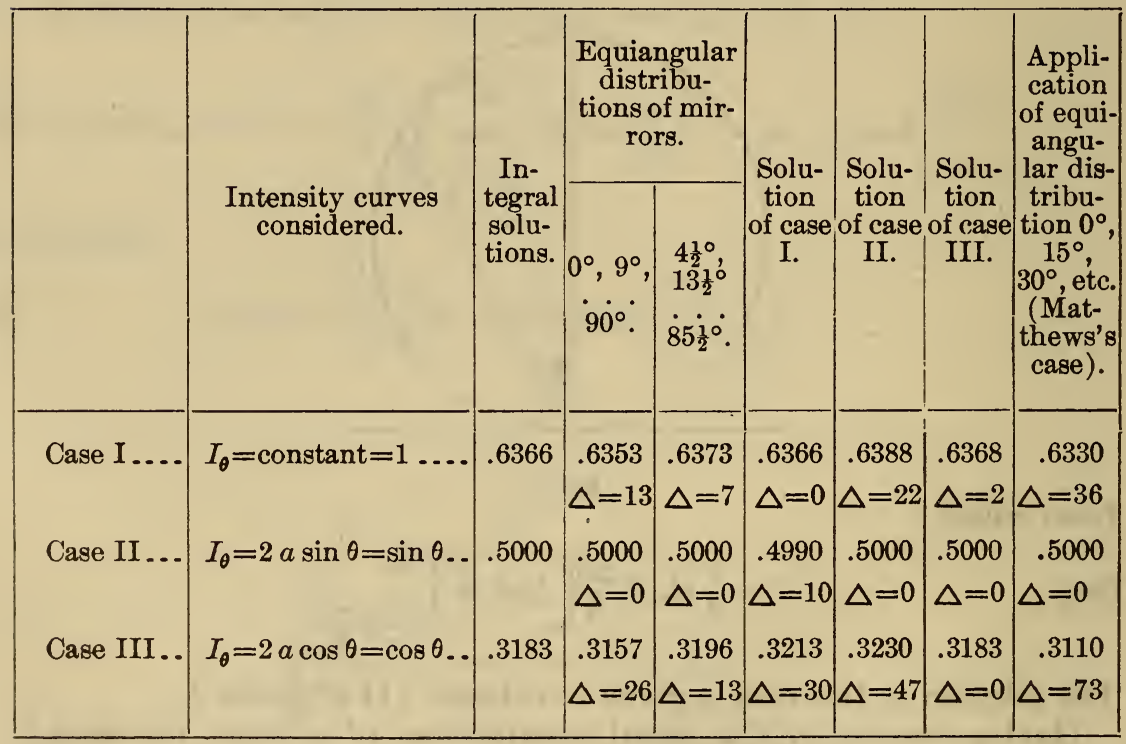

In Table II are given the results of applying the five different distributions to each of the three intensity curves considered. Column 
II contains the equations of the intensity curves of the different cases; column III contains the true integral values; columns IV and V give the results of applying the two equal distributions to each of the three cases; columns VI, VII, and VIII give the results of applying the distributions of cases I, II, and III to each of the three cases; and, finally, column IX gives the results of applying the equiangular arrangement of eleven mirrors to each of the three cases, in order to see how accurately the Matthews photometer, as ordinarily constructed, gives the mean spherical value. In each column the differences, $\Delta$, between the values found and the true integral values are given.

From a consideration of Table II it seems clear that the distribution of mirrors corresponding to case III satisfies all the cases most completely, the greatest error being well under 0.1 per cent. The equal distribution $4 \frac{1}{2}{ }^{\circ}, 13 \frac{1}{2} \circ, \ldots .175 \frac{1}{2}^{\circ}$, however, is very satisfactory, the greatest error being about $\frac{1}{2}$ per cent. The equal distribution of eleven mirrors every $15^{\circ}\left(0^{\circ}, 15^{\circ}, 30^{\circ}, \ldots 180^{\circ}\right)$, may give errors amounting to over 2 per cent.

It is impossible, of course, to take into consideration all the actual forms of intensity curves existing, but it would seem that a distribution of mirrors which will satisfy the three typical curves considered would also satisfy any of the ordinary forms of curves with which we are familiar in the photometry of such sources as incandescent lamps. For example, the empirical curve of the "downward light" lamp coincides with the ellipse

$$
\frac{x^{2}}{a^{2}}+\frac{y^{2}}{(8.2 a)^{2}}=1
$$

so closely that it is scarcely possible to detect any difference between the two curves platted to the scale of $a=4 \mathrm{~cm}$. Now the true integral value for the above ellipse is 1.722 , whereas the value obtained by applying the distribution of case III comes out 1.723 .

Thus we see that twenty mirror-pairs properly distributed are entirely sufficient theoretically to give mean spherical, and at the same time mean hemispherical candle-power values to within an error well under that which could be detected in making a setting under the most favorable conditions.

\section{General Theory of the Russeli-Léonard Photometer.}

As before, let

$$
I_{\theta}=f(\theta)
$$

be the equation of the intensity curve in any vertical plane through the source, assuming the surface of distribution of intensity to be one $5834-$ No. $2-05-10$ 
of revolution around the vertical axis, which is taken as the axis of the lamp in every case.

Then the flux across any zone, $d \theta$, of the unit sphere is,

$$
L_{z}=2 \pi I_{\theta} \sin \theta d \theta
$$

the total flux is,

$$
L_{s}=\int_{0}^{\pi} 2 \pi I_{\theta} \sin \theta d \theta
$$

and hence the mean spherical intensity is,

$$
I_{m s}=\frac{L_{s}}{4 \pi}=\frac{1}{2} \int_{0}^{\pi} I_{\theta} \sin \theta d \theta
$$

Similarly, the mean hemispherical intensity is,

$$
\begin{aligned}
I_{m h s} & =\frac{1}{2 \pi} \int_{0}^{\frac{\pi}{2}} 2 \pi I_{\theta} \sin \theta d \theta \\
& =\int_{0}^{\frac{\pi}{2}} I_{\theta} \sin \theta d \theta
\end{aligned}
$$

or, in general, the mean intensity for any zone $\theta_{m-1}$ to $\theta_{m}$ is,

$$
\begin{aligned}
I_{m z}= & \frac{\int_{\theta_{m-1}}^{\theta_{m}} 2 \pi I_{\theta} \sin \theta d \theta}{\int_{\theta_{m-1}}^{\theta_{m}} 2 \pi \sin \theta d \theta}=\frac{\int_{\theta_{m-1}}^{\theta_{m}} I_{\theta} \sin \theta d \theta}{[\cos \theta]_{\theta_{m}}^{\theta_{m-1}}} \\
& =\frac{1}{\cos \theta_{m-1}-\cos \theta_{m}} \int_{\theta_{m-1}}^{\theta_{m}} \sin \theta d \theta
\end{aligned}
$$

If, now, we divide the surface of the sphere into $n$ zones of equal area, the mean spherical intensity is given by the equation

$$
I_{m s}=\frac{1}{n} \sum_{m=1}^{m=n} I_{m z}=\frac{1}{n} \Sigma_{1}^{n} \frac{1}{\cos \theta_{m-1}-\cos \theta_{m}} \int_{\theta_{m-1}}^{\theta_{m}} I_{\theta} \sin \theta d \theta
$$

in which $m$ indicates the order of the zone, counting from $\theta=0^{\circ}$, and the limits of integration for the several partial integrals are the angles corresponding to the circles of division between the successive zones. 
Suppose, now, that we place a mirror in each of the $n$ zones and let the light from these mirrors fall directly upon the photometer screen. It is evident that the illumination produced on the screen is $n$ times that which would be produced by a source having an intensity in the direction of the screen equal to the mean spherical intensity of the lamp under test, assuming that the summational value differs from the true integral value of the mean spherical intensity by a negligibly small error. This error, however, depends greatly of course, as we saw above, upon the form of the intensity curve, but just as in the theory of the Matthews's instrument, for any one simple form of curve assurned, it is not difficult to determine the position of each mirror in its respective zone, so that the intensity given by the mirror is exactly equal to the mean intensity of the zone in which it lies.

Thus, if we know the intensity curve

$$
I_{\theta}=f(\theta)
$$

we determine $\theta_{m}^{1}$ for each zone, so that

$$
I_{m z}=f\left(\theta_{m}^{1}\right)=\frac{1}{\cos \theta_{m-1}-\cos \theta_{m}} \int_{\theta_{m-1}}^{\theta_{m}} f(\theta) \sin \theta d \theta
$$

Then the intensity at each angle, $\theta_{m}^{1}$, will be the mean intensity for the respective zone.

If, now, we place a mirror at each angle, $\theta_{m}^{1}$, as determined from the previous equation, assuming the form of intensity curve, then the mean value of the several intensities as given by the various mirrors will exactly equal the mean spherical intensity of the source, provided, of course, that the source has the same form of intensity curve as was assumed in the calculation of the various angles, $\theta_{m}^{1}$.

IV. Application of the Russell-Léonard Photometer to Arc LAMPS.

As the Russell-Léonard photometer is of the same general form as the Matthews arc lamp instrument, with the important point of difference that in the former instrument the objectionable smoked disk of the Matthews photometer is eliminated, we shall briefly consider first the distribution of mirrors which must be made in the Russell-Léonard photometer, in order that in the photometry of arc lamps it may yield a summational result agreeing as nearly as possible with the true integral value.

We must first decide upon the dimensions of the instrument to be designed, the size and number of the mirrors, etc., which will depend to a great extent upon the accuracy that we desire to cbtain. We then 
divide the sphere into the number of zones decided upon, determine the limiting angles between the successive zones, and with the use of eq. (28) calculate the angle, $\theta_{m}^{\prime}$, for each zone corresponding to the mean value of the intensity for that zone, upon the assumption of the polar equation of the intensity curve of the arc. Though this equation is not the same for different kinds of arc lamps, or even for different vertical planes of the same arc at any one time, yet we can assume a hypothetical curve which will correspond more or less closely with the different curves met with, since all the curves for arc lamps are of the same general type, with a maximum at about $\theta=45^{\circ}$ or $50^{\circ}$ and falling off rapidly toward $\theta=0^{\circ}$ and $\theta=90^{\circ}$. If the arc is surrounded by a globe that scatters the light, the curve approaches more nearly to a circle with the source at the center, and since any arrangement of mirrors within the arcs of equal area will necessarily give absolutely the true result for a source having the same intensity in all directions, a distribution of mirrors which will satisfy any definite intensity curve will not yield very large errors if that curve is made to approach a circle with the source at the center. Hence, if we were to take the typical curve for a direct-current open arc, and determine the mirror arrangement satisfying this case, the errors that would arise on using the instrument for the inclosed arc would be relatively small; and, moreover, since the instrument is symmetrical with respect to the horizontal direction, it would be equally applicable to the alternatingcurrent arc, whose intensity curve is approximately the same as that of the direct-current lamp, except that the distribution in the lower hemisphere is repeated in the upper one.

Let us then, as a first approximation, assume that the intensity curve is an ellipse with the major axis inclined at $45^{\circ}$ to the vertical, and with one end of the major axis coincident with the source (fig. 4).

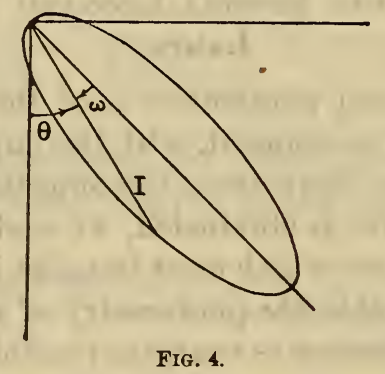

The equation, expressed in rectangular coordinates, is

$$
\frac{(x-a)^{2}}{a^{8}}+\frac{y^{2}}{b^{2}}=1
$$


Now the mean intensity for any zone, $\theta_{m-1}-\theta_{m}$, is given by eq. (26),

$$
I_{m z}=\frac{1}{\cos \theta_{m-1}-\cos \theta_{\dot{m}}} \int_{\theta_{m-1}}^{\theta_{m}} I_{\theta} \sin \theta d \theta
$$

If we make $I_{\theta}$ equal to the radius vector of the ellipse given by eq. (29), we get, on transforming to the variable, $\omega$,

(30) $I_{m z}=\frac{a b^{2} \sqrt{2}}{\cos \left(45^{\circ}-\omega_{m-1}\right)-\cos \left(45^{\circ}-\omega_{m}\right)} \int_{\omega_{m-1}}^{\omega_{m}} \frac{\cos ^{2} \omega-\sin \omega \cos \omega}{b^{2} \cos ^{2} \omega+a^{2} \sin ^{2} \omega} d \omega$ which on integration becomes

(31) $I_{m z}=\frac{a b^{2} \sqrt{2}}{\left[a^{2}-b^{2}\right]\left[\cos \left(45^{\circ}-\omega_{m-1}\right)-\cos \left(45^{\circ}-\omega_{m}\right)\right]}\left[\frac{a}{b} \tan ^{-1} \frac{a}{b} \tan \omega-\right.$

$$
\left.\omega-\frac{1}{2} \log \left\{\left(a^{2}-b^{2}\right) \sin ^{2} \omega+b^{2}\right\}\right]_{\omega_{m-1}}^{\omega_{m}}
$$

If, now, we determine upon eight zones, the limiting values of $\theta$ are obtained by evaluation of the equations

$$
\begin{aligned}
& \theta_{0}=\cos ^{-1} 1=0^{\circ} \\
& \theta_{1}=\cos ^{-1} \frac{3}{4}=41^{\circ} 25^{\prime} \\
& \theta_{2}=\cos ^{-1} \frac{1}{2}=60^{\circ} \\
& \theta_{3}=\cos ^{-1} \frac{1}{4}=75^{\circ} 31^{\prime} \\
& \theta_{4}=\cos ^{-1} 0=90^{\circ}
\end{aligned}
$$

from which the limiting values of $\omega$ can be calculated from the equation

$$
\omega=45^{\circ}-\theta
$$

Moreover for eight zones

$$
\cos \theta_{m-1}-\cos \theta_{m}=\cos \left(45^{\circ}-\omega_{m-1}\right)-\cos \left(45^{\circ}-\omega_{m}\right)=\frac{1}{4}
$$

If, now, we take the semimajor axis equal to unity, and the semiminor axis equal to one-third,--a ratio quite closely in accord with observation-we obtain

$$
I_{m z}=\frac{\sqrt{2}}{2}\left[3 \tan ^{-1} 3 \tan \omega-\omega-\frac{1}{2} \log \frac{1}{9}\left\{8 \sin ^{2} \omega+1\right\}\right]_{\omega_{m-1}}^{\omega_{m}}
$$

If we evaluate this expression for each of the eight zones, or, since there is perfect symmetry with respect to the horizontal axis, for each 
of four zones, and then substitute successively the values obtained in the equation

$$
\theta_{m z}=45^{\circ} \cdot \cos ^{-1} \frac{1}{8 I_{m z}}\left(\sqrt{72 I_{m z}^{2}+1}-1\right)
$$

which is easily deduced from the equation of the ellipse, putting $I_{m z}$ for the radius vector, we get as the angles to which the mean radii vectores of the several zones of equal area correspond

$$
\begin{aligned}
& \theta_{1}^{\prime}=28^{\circ} 51^{\prime} \\
& \theta_{2}^{\prime}=52^{\circ} 33^{\prime} \\
& \theta_{3}{ }^{\prime}=67^{\circ} 23^{\prime} \\
& \theta_{4}^{\prime}=82^{\circ} 20^{\prime}
\end{aligned}
$$

If, now, we were to place a mirror at each of these angles, both above and below the horizontal axis, but only on one side of the vertical diameter, then for a source having an intensity curve identical with the assumed ellipse, the mean value of the intensity from the eight mirrors, obtained by dividing the sum of the intensities from the mirrors by eight, would exactly equal the mean spherical intensity of the source, provided, of course, that the surface of distribution of intensity is a figure of revolution around the vertical axis. This is never the case, and since, in general, when the maximum curve is on one side of the arc the minimum curve is on the other side, by placing eight other mirrors on the other side of the vertical diameter at the same angles as those already located, the mean intensity in the complete vertical plane is obtained, which will be more nearly equal to the mean spherical value than the value obtained from mirrors on one side of the vertical diameter only. In order to obtain mean spherical values of any considerable accuracy the mean of a great number of determinations of the mean vertical intensity must be taken.

In a similar manner mean hemispherical determinations can be made if the surface of the sphere is divided into an even number of zones, as was pointed out above in the discussion of the Matthews instrument.

The typical curve of the open arc does not correspond exactly with such an ellipse as assumed in the preceding discussion, but the errors in arc-light photometry are in other respects necessarily so large that the difference between the true mean value for a vertical plane and that given by the mirrors arranged to satisfy the ellipse distribution will in general be much smaller than the other errors incident to the measurement. If a closer agreement between the integral and summational values of the arc is desired without increasing the number of mirrors, it would be necessary to investigate in detail the ty pical curve 
of the arc, and from this curve to compute the angles corresponding to the true distribution of mirrors.

In order to determine the magnitude of the error that would arise if the form of intensity curve differed to any extent from an ellipse, the distribution of mirrors satisfying the ellipse was applied to the two curves,

$$
\begin{aligned}
& I_{\theta}=\sin \theta \\
& I_{\theta}=\cos \theta
\end{aligned}
$$

The true integral means of these are, respectively, [eq. (24)]

$$
\begin{gathered}
I_{m s}=\frac{1}{2} \int_{0}^{\pi} I_{\theta} \sin \theta d \theta=\int_{0}^{\frac{\pi}{2}} \sin ^{2} \theta d \theta=.7854 \\
I_{m s}=\frac{1}{2} \int_{0}^{\pi} I_{\theta} \sin \theta d \theta=\int_{0}^{\frac{\pi}{2}} \sin \theta \cos \theta d \theta=.5000
\end{gathered}
$$

whereas the values given by one-fourth of the summations of the sines and cosines, respectively, of the angles of eq. (37) are

$$
\begin{aligned}
& I_{m s}=.7976 \\
& I_{m s}=.5005
\end{aligned}
$$

which are in error by 1.6 per cent and 0.1 per cent, respectively.

\section{Application of the Russell-Léonard Photometer to INCANDESCENT LAMPS.}

In the application of the Russell-Léonard photometer to incandescent lamps the problem is just the same as in the application to are lamps, except that here we seek the distribution of mirrors that will satisfy the three intensity curves

$\begin{array}{ll}\text { Case I } & I_{\theta}=\text { constant } \\ \text { Case II } & I_{\theta}=\sin \theta \\ \text { Case III } & I_{\theta}=\cos \theta\end{array}$

Any distribution of mirrors which will satisfy these three cases will yield very small errors when applied to any actual curves. Moreover, since any distribution of mirrors in zones of equal area will satisfy case I, we simply have to determine the distribution most nearly satisfying cases II and III. 
The true integral values for cases II and III, respectively, are [eqs. $(40)$ and (41)]

$$
\begin{aligned}
& I_{m s}=.7854 \\
& I_{m s}=.5000
\end{aligned}
$$

If we divide the surface of the sphere into twelve zones instead of eight, the distribution of mirrors satisfying case II, as determined by evaluation of eq. (28) for each of the twelve zones, after putting for $f(\theta)$ its value $\sin \theta$, gives an error of 1.0 per cent when applied to case III. Similarly the true distribution for case III gives an error of 0.7 per cent when applied to case II. If, however, we take a distribution of mirrors intermediate between the two true distributions, i. e., if we take

$$
\begin{aligned}
& \theta_{1}=22^{\circ} 47^{\prime} \\
& \theta_{2}=41^{\circ} 14^{\prime} \\
& \theta_{3}=54^{\circ} 15^{\prime} \\
& \theta_{4}=65^{\circ} 14^{\prime} \\
& \theta_{5}=75^{\circ} 20^{\prime} \\
& \theta_{6}=85^{\circ} \quad 8^{\prime}
\end{aligned}
$$

the error is divided between the two cases, so that the summational values for cases II and III are, respectively,

$$
\begin{aligned}
& I_{m s}=.7883 \\
& I_{m s}=.5025
\end{aligned}
$$

the corresponding percentage errors being 0.4 per cent and 0.5 per cent.

We have divided the surface of the sphere into 12 zones in this case, as compared with 8 in dealing with arc lamps, because in the first place a higher accuracy is obtainable in the photometry of incandescent lamps, and, secondly, if the incandescent lamp is rotated, 12 zones demand only 12 mirrors which can be arranged around the whole circle, whereas 8 zones demand 16 mirrors in the arc-light photometer, -8 mirrors on each side of the vertical diameter. Thus, for incandescent lamp work, 12 mirrors, each 6 inches broad, can be arranged in a circle of 3 feet diameter, and yield a result accurate to within about 0.5 per cent for lamps of various intensity curves, whereas the Matthews instrument, as at present constructed, gives an error of over 2 per cent (Tab'e II) when applied to the lamp having the intensity curve $I_{\theta}=\cos \theta$.

It has not been the intention, in this discussion of the RussellLéonard photometer, to make the treatment exhaustive, but rather to suggest the method which can be applied to locate the mirrors in the zones after the uses to which the instrument is to be put and the consequent general dimensions of the instrument have been determined. 



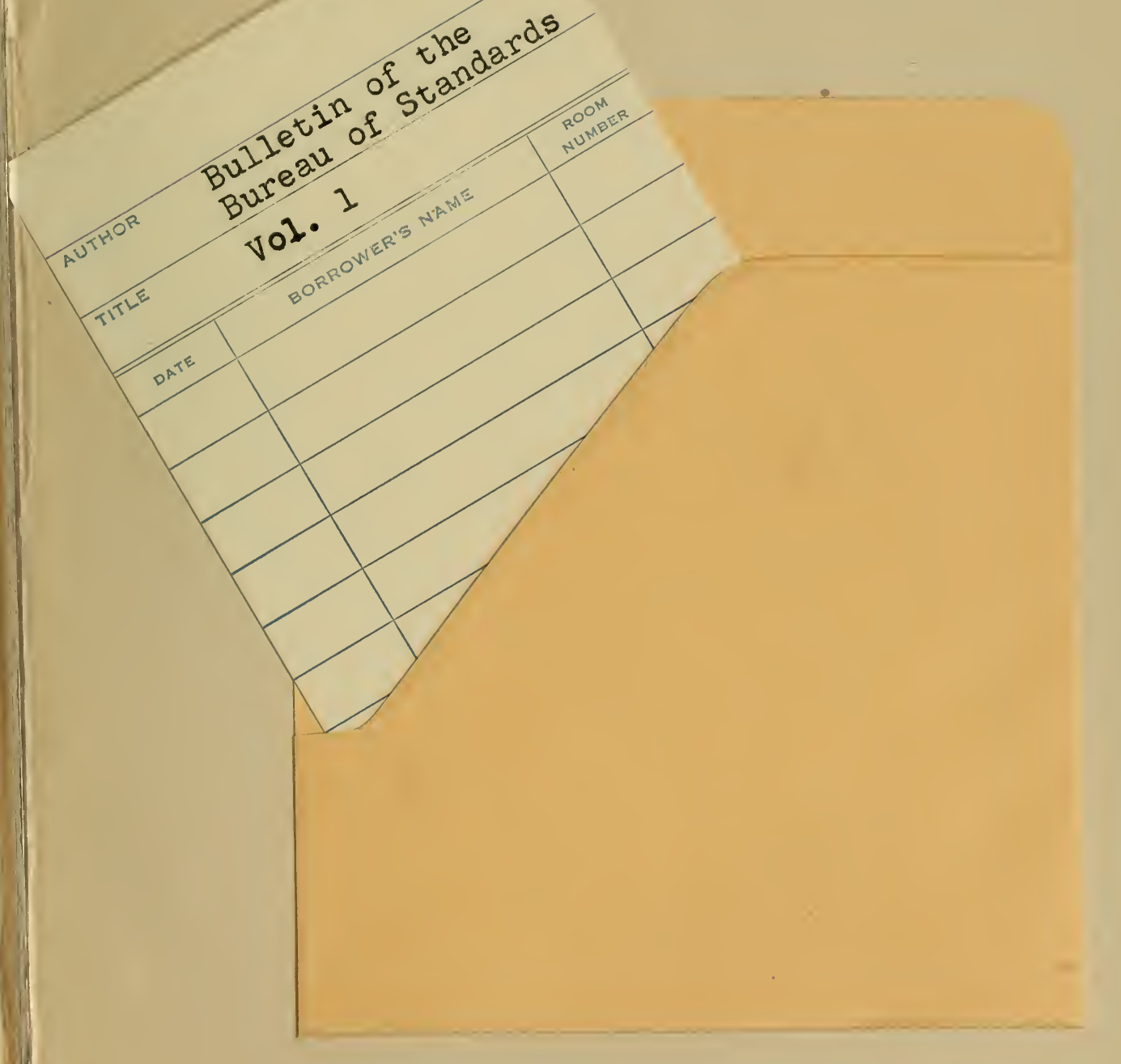


